\title{
NORMALIZACION ESPAÑOLA SOBRE CENIZAS VOLANTES (NORMAS UNE)
}

Antonio Pardo, Dr. en Ciencias Quimicas Asociación Española de Fabricantes de Hormigón Preparado (ANEFHOP)
'M. Pilar de Luxán, Dra en Ciencias Químicas Instituto de la Construcción y del Cemento

"Eduardo Torroja" (CSIC)

\section{RESUMEN}

En 1983 se creó el Grupo de Trabajo "Adiciones al Hormigón" en el seno de la Comisión Técnica-83 "Hormigones"/IRANOR, actualmente transferido a AENOR.

Su finalidad principal, durante estos años, ha sido la elaboración de la normativa española sobre las cenizas volantes que hayan de ser utilizadas en morteros y hormigones de cemento portland. Su exposición es el objeto principal de este trabajo, en el que se incluye también un análisis comparativo con la normativa internacional, así como los pasos a seguir en la actualización constante de las normas.

Asimismo se recoge un resumen de las caracteristicas de las cenizas volantes generadas en España con anterioridad a la elaboración de las Normas UNE.

\section{SUMMARY}

The "Concrete Additives" task force was created within the "Concretes"/IRANOR Technical Commission-83 in 1983. It presently answers to the AENOR.

Its principle objective during these years has been the elaboration of Spanish regulations concerning fly ash to be used in morters and concretes made with Portland cement. The main purpose of this study, in which a comparative analysis of international regulations is also included, is the exposition of these regulations, as well as the steps to be taken in their constant up-dating.

In addition, a summary of the characteristics of fly ash produced in Spain before the elaboration of the UNE norms is also included.

\section{INTRODUCCION}

Desde los primeros años de la década de los ochenta se ha venido constatando la utilización de cenizas volantes (c.v.) como adición a los hormigones en centrales de hormigón y en hormigones fabricados a pie de obra.

Estas adiciones ya se venían utilizando para obras determinadas, desde años atrás, en países como E.E.U.U., Alemania, etc., por considerarse ventajosa su utilización, fundamentalmente por la disminución del desprendimiento del calor de hidrátación en grandes obras de hormigón en masa, como son las presas de fábrica, obras en las que las prestaciones mecánicas de los hormigones tienen una baja incidencia.
Las particularidades por las que atraviesa la utilización de las c.v., actualmente en España, son semejantes a las de otros países europeos, y parten del deseo de utilizar al máximo la elevada producción de c.v., unido a la consideración, tanto de las mejoras de algunas de las características que confieren a los morteros y hormigones en los que se incorporan, como de la disminución de los costes y el mantenimiento de las exigencias de prestaciones mecánicas, protección de las armaduras, durabilidad, etcétera.

No obstante, desde el punto de vista de la calidad, se hace preciso especificar una serie de valores para una gama de parámetros en las cenizas volantes, que permitan garantizar las características de los morteros u hormigones en los que vayan a ser adicionadas. 


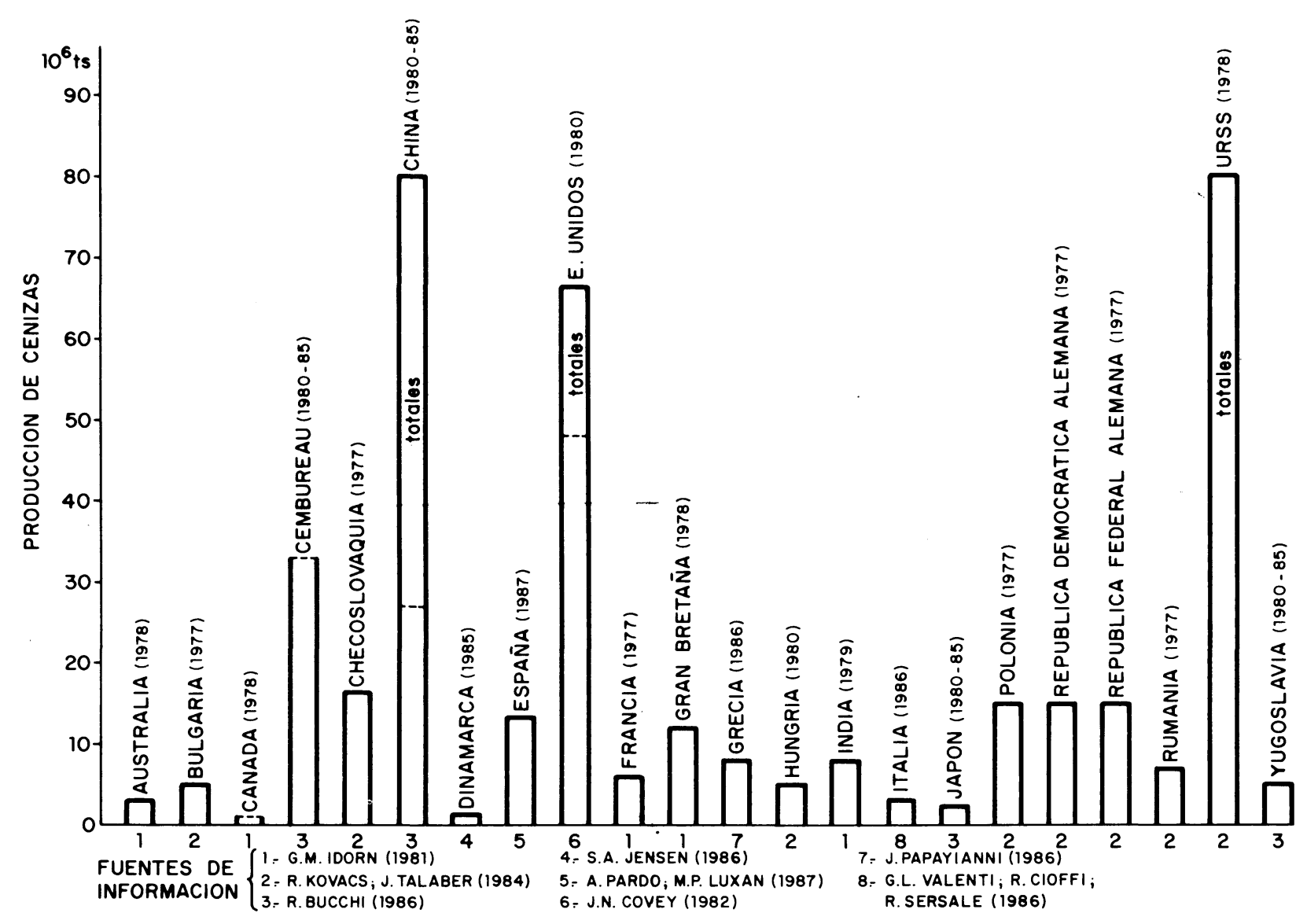

Fig. 1.-Panorámica mundial de producción de cenizas.

Así, por ejemplo, a un hormigón con adición de c.v. que, desde el punto de vista económico, puede competir a igualdad de características con un hormigón sin este tipo de adición, es preciso exigirle que en unas condiciones normalizadas posea una determinada actividad resistente. Otros factores a tener en cuenta al adicionar cenizas volantes son que éstas no puedan presentar potencialmente inestabilidad en los hormigones, y también conocer las variaciones sobre la demanda de agua necesaria para conseguir una determinada consistencia que puede ser alterada por su empleo, etcétera.

Entre los aspectos químicos que se deben considerar, se encuentra el contenido máximo de $\mathrm{SO}_{3}$, en razón a evitar posibles problemas de durabilidad posteriores y las pérdidas máximas por calcinación, en razón sobre todo a la utilización de aditivos en los hormigones por su relación con el contenido de inquemados de las cenizas.

Estas características, entre otras, se han tenido en cuenta a la hora de establecer en España la normativa para la utilización de c.v. como adición a los hormigones de cemento portland, y que a lo largo de los apartados de este trabajo se irá exponiendo.

En los inicios del desarrollo de la normativa sobre estos materiales se ha seguido el criterio de que las nor- mas tengan un sello conservador y aparezcan como experimentales durante el período de un año, ya que la experiencia relativa a los hormigones con c..., especialmente a largo plazo, no es grande y no resulta aconsejable trasplantar íntegramente la de otros paises, cuyas materias primas, carbones y utilizaciones pueden ser radicalmente distintas.

En la Fig. 1 se expone una panorámica mundial de la producción de cenizas, cuyos datos se refieren a estos últimos años y se han recogido de numerosas fuentes (1), (2), (3), (4), (5), (6) y (7). En España la producción de c.v. asciende a cerca de 12 millones de toneladas al año.

\section{CARACTERISTICAS DE LAS CENIZAS VOLANTES}

La formación de las cenizas volantes está condicionada por el proceso térmico de la central termoeléctrica (temperatura, (1), ...), tipo de carbón (composición, granulometria,...), etc. y es determinante, a su vez, de sus características físicas, químicas, mineralógicas y morfológicas y, por lo tanto, de su comportamiento posterior.

Las cenizas volantes no son un material homogéneo, ya que el conjunto de partículas que la constituyen difie- 
ren en su composición química, tamaño, granulometría, textura superficial, ...

Durante el proceso de combustión y dependiendo de la temperatura alcanzada, transcurren un gran número de reacciones químicas: se produce la deshidratación de los minerales asociados al carbón, los carbonatos se des. componen, las fases minerales cambian o llegan a la fusión, el yeso se deshidrata y las piritas se oxidan.

Cuanto más elevada sea la temperatura de combustión, mayor es la cantidad de material fundido; así, mientras que con una temperatura de $1.000^{\circ} \mathrm{C}$ el porcentaje alcanza sólo el $20 \%$ del total, con temperaturas entre 1.500 y $1.700{ }^{\circ} \mathrm{C}$, todas las fases minerales se encuentran ya fundidas (1).

La naturaleza vitrea o cristalina de las cenizas volantes que se originan está condicionada, no sólo a la temperatura de combustión alcanzada, sino a la velocidad de enfriamiento. Un enfriamiento rápido favorece que la proporción entre el contenido de material vítreo y cristalino aumente, con la consiguiente influencia en la reactividad del material y en sus propiedades puzolánicas.

Las partículas gruesas caen al fondo de la caldera y las partículas más finas permanecen suspendidas en los gases, adquiriendo forma esférica debido a la tensión superficial; éstas son las que toman el nombre de cenizas volantes.

El examen al microscopio de las cenizas volantes muestra multitud de pequeños granos de diferente tamaño, forma (esferas predominantemente), composición y textura superficial. Existen esferas huecas que albergan en su interior otras más pequeñas o bien partículas de inquemados, otras son compactas, otras porosas. Junto a ellas hay partículas irregulares que consisten en cuarzo o fragmentos vítreos e inquemados (8).

\subsection{Cenizas volantes procedentes de centrales termoeléctricas españolas}

Las centrales termoeléctricas españolas se encuentran diseminadas en todo el territorio; predominan en la zona norte, en lugares donde se localizan minas de carbón.

Los últimos trabajos realizados, sobre las cenizas volantes españolas, ponen de manifiesto la semejanza de sus caracteristicas con las procedentes de centrales termoeléctricas de otros países (9).

Los estudios sobre las cenizas volantes son muy complejos, ya que cada partícula que las constituye es diferente en composición química, estructura interna y morfología. La caracterización se hace sobre el conjunto del material y dependerá de la variabilidad de las diferentes partículas.

El contenido vitreo, la textura superficial y la morfología de las partículas son factores que tienen una importancia primordial en el comportamiento del material. Su estudio entraña gran dificultad, aunque existen interesantes aportaciones como ha hecho recientemente el profesor S. Diamond (10).

Para penetrar en el conocimiento de estos materiales hay que considerar también tres aspectos fundamentales: características químicas, mineralógicas y físicas:

- EI ANALISIS QUIMICO de las cenizas volantes españolas proporciona una suma de óxidos principales $\left(\mathrm{SiO}_{2}+\mathrm{Al}_{2} \mathrm{O}_{3}+\mathrm{Fe}_{2} \mathrm{O}_{3}\right)$ superior al $75 \%$; el contenido en $\mathrm{SO}_{3}$ es variable, depende de la composición del carbón original, pero sus valores son inferiores al 5 por ciento. La pérdida por calcinación oscila mucho, refleja el contenido de inquemados que posee la ceniza. El porcentaje de $\mathrm{MgO}$ es inferior al 2,5 por ciento (9).

El contenido de cal de las cenizas volantes españolas es muy variable. P. K. Mehta (11) estableció una clasificación de las c.v. respecto a su contenido en cal, denominándolas: c.v. altas en cal y c.v. bajas en cal, basándose en que al encuadrar las c.v. en cada uno de estos grupos, su composición mineralógica presentaba compuestos análogos.

En la COMPOSICION MINERALOGICA de las C.V. españolas se han encontrado compuestos que aparecen en las C.V. de otros países (9), (12).

En la Fig. 2 de la página siguiente se exponen, como ejemplo, los difractogramas de rayos $X$ de dos cenizas volantes españolas: a) baja en cal y b) alta en cal.

Las cenizas volantes españolas clasificadas como bajas en cal presentan los siguientes compuestos mineralógicos: cuarzo $(Q)$, mullita $(M)$, hematites $(H)$, calcita(C), aluminatos cálcicos, etc. Las C.V. altas en cal son más complejas y tienen mayor número de compuestos mineralógicos; en el estudio de las c.v. españolas se han encontrado: cuarzo $(Q)$, hematites $(H)$ y anhidrita $(A n)$; en ocasiones se observa la presencia de silicatos cálcicos (CS), portlandita $(\mathrm{P})$, cal libre $(C)$ y sílicoaluminatos cálcicos (13).

- La DISTRIBUCION GRANULOMETRICA de las partículas de las cenizas volantes, es una de las características más variables; en ella influyen fundamentalmente el grado de pulverización del carbón de origen, el proceso térmico (temperatura alcanzada, contenido de inquemados, ...) y el tipo de precipitadores de la central.

Las c.v. españolas presentan curvas de distribución del tamaño de partículas análogas a las procedentes de centrales termoeléctricas de otros países (9), (14). 

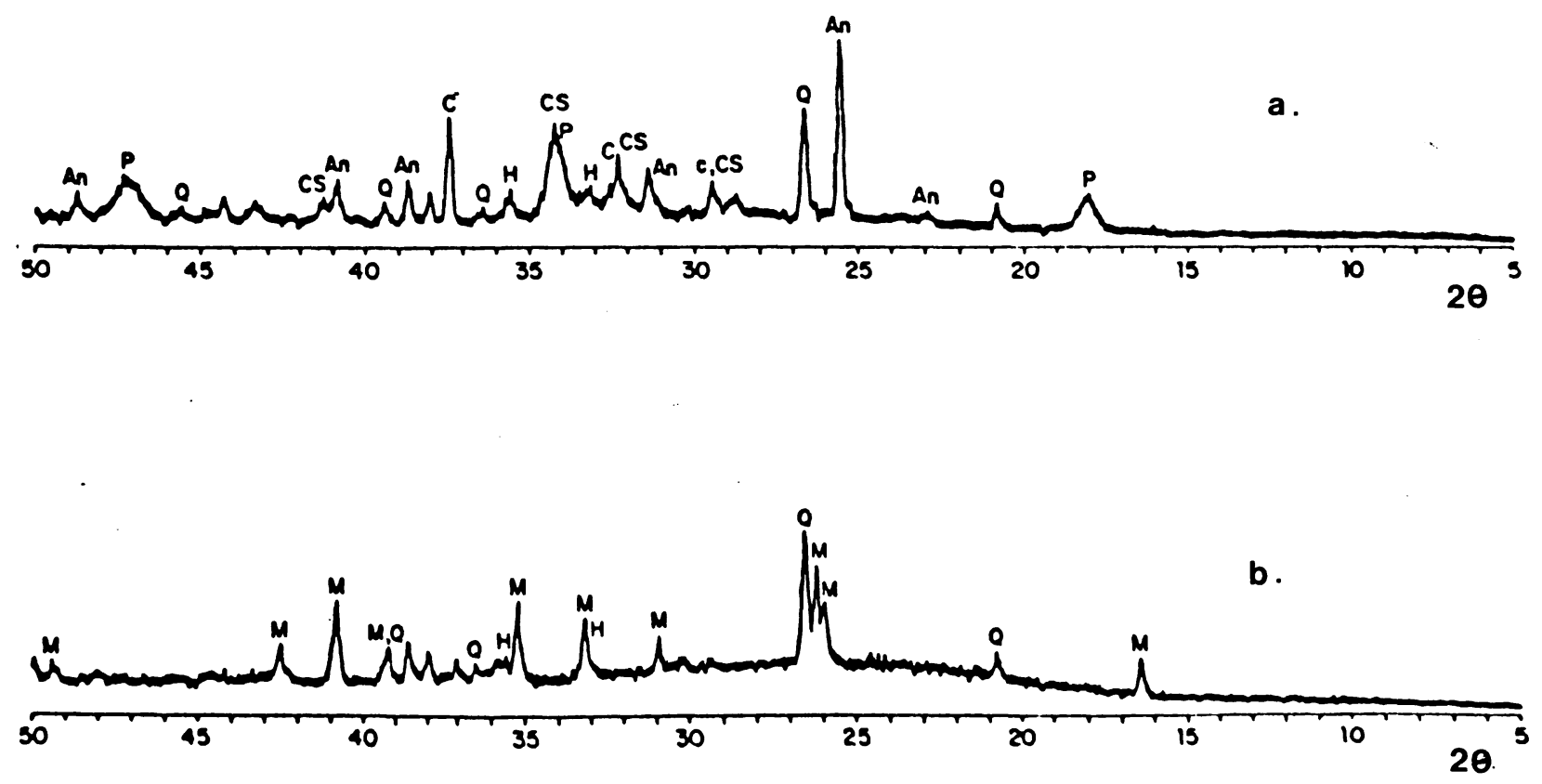

Fig. 2.-Difractogramas de rayos $X$ de cenizas volantes españolas:

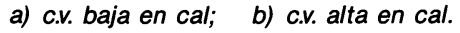

\section{NORMALIZACION DE LAS CENIZAS VOLANTES PARA SU USO EN EL HORMIGON}

La necesidad de la existencia de normas para un buen uso de las cenizas volantes en cualquiera de sus aplicaciones es un hecho, especialmente si se tiene en cuenta que no todas las c.v. se prestan a todos los empleos, de forma que para cada caso particular se habrán de utilizar atendiendo conjuntamente a sus propiedades y a las necesidades técnicas concretas.

Al plantearse el diseño de la normativa sobre c.v. hay que apoyarse en el conocimiento de sus características, que dependen primordialmente de su génesis, y que condicionarán su comportamiento posterior.

La complejidad del problema del origen y las causas de la reactividad de las c.v. hace que muchos factores principales que determinan sus propiedades puzolánicas, entre otras, no se puedan normalizar por las dificultades que entrañaria su cuantificación (textura superficial, etcétera).

Además, las especificaciones que se recojan en las normas deben ser seleccionadas considerando muchos aspectos, inclusive aquellos que permitan la valoración del material para las prestaciones que se busquen en él, como por ejemplo la sencillez y facilidad de medida en las centrales de hormigón.
La normalización tiene, por tanto, sus propias limitaciones.

Anteriormente se han recogido brevemente las características de las cenizas volantes españolas, para resaltar el hecho de su analogía con las procedentes de otros países. La consecuencia inmediata es que la normalización española sobre cenizas volantes habrá de ser también similar en sus especificaciones a las ya existentes en el extranjero.

A la hora de establecer la normativa sobre c.v. para su uso en hormigón, hay que considerar dos aspectos fundamentales: por una parte, las características que se deben exigir al material en cuanto a su calidad, en su recepción; y, por otra, aquellas que van a condicionar las propiedades del hormigón resultante.

Con objeto de hacer una exposición más simplificada de las normas elaboradas en España sobre c.v., se presenta un esquema que se recoge en la Tabla l, donde se incluyen las especificaciones relativas a las características quimicas y físicas, asi como el valor fijado correspondiente a la Norma UNE 83 415, y los métodos de ensayo para su determinación, con la reseña de la norma UNE donde quedan descritos. 
Tabla I

Adiciones al hormigón.

Cenizas volantes. Caracterización según Norma UNE 83415.

\begin{tabular}{|c|c|c|c|c|c|}
\hline \multicolumn{3}{|c|}{ DETERMINACION } & $\begin{array}{l}\text { METODO DE } \\
\text { ENSAYO } \\
\text { NORMA UNE NQ }\end{array}$ & $\begin{array}{l}\text { ESPECFICACION } \\
\text { UNE } 83415\end{array}$ & ENSAYO \\
\hline \multirow{3}{*}{ 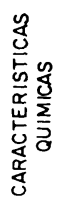 } & \multicolumn{2}{|c|}{ Humedad (\%) } & 83431 & máx. 1,5 & $\begin{array}{l}\text { En estufa } \\
T=105 \pm 5^{\circ} \mathrm{C} \\
\text { tiempo }=2 \text { horas }\end{array}$ \\
\hline & \multicolumn{2}{|c|}{$\mathrm{SO}_{3}(\%)$} & 83432 & móx.4,5 & $\begin{array}{l}\text { Método gravimétrico } \\
\text { (Precipitación de } \mathrm{BaSO}_{4} \text { ) }\end{array}$ \\
\hline & \multicolumn{2}{|c|}{$\begin{array}{c}\text { P.P. } \\
\text { Colcinación }(\%)\end{array}$} & 83433 & móx. 6 & $\begin{array}{l}\text { En horno eléctrico } \\
T=750 \pm 50^{\circ} \mathrm{C} \\
\text { tiempo }=1 \text { horo }\end{array}$ \\
\hline \multirow{4}{*}{ 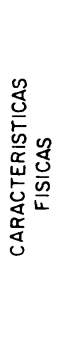 } & $\begin{array}{l}\text { Finura } \\
(\%)\end{array}$ & & 83450 & $\begin{array}{l}\text { máx. } 40 \\
\text { máx. } 15\end{array}$ & Tamizado en húmedo \\
\hline & \begin{tabular}{|} 
Actividod \\
Resistente \\
$(\%)$
\end{tabular} & $\begin{array}{c}28 \\
\text { dios } \\
90 \\
\text { dios }\end{array}$ & 83451 & $\begin{array}{l}\geq 75 \\
\geq 90\end{array}$ & $\begin{array}{l}\text { Mortero normal } 1 / 3 \text { de } \\
\text { ceniza volante ( } 30 \% \text { peso) } \\
\text { con cemento portland ( } 70 \% \text { pe } \\
\text { so)y relación con mortero control }\end{array}$ \\
\hline & \multicolumn{2}{|c|}{ Demango ge agua } & 83452 & máx.100 & $\begin{array}{l}\text { Mortero normal y mortero } \\
\text { control idem a } 83451\end{array}$ \\
\hline & \multicolumn{2}{|c|}{$\begin{array}{l}\text { Expansión Agujos } \\
\text { Le Chatelier } \\
(\mathrm{mm})\end{array}$} & 83453 & máx.10 & $\begin{array}{l}\text { Estabilidad en volumen de ung } \\
\text { ceniza volante ( } 30 \% \text { peso)con } \\
\text { cemento portland ( } 70 \% \text { peso) }\end{array}$ \\
\hline
\end{tabular}

En la Tabla II se contempla una relación de las normas que figuran en UNE 83415.

Todas las normas se han publicado como experimentales durante el período de un año, desde el principio de su aparición en 1987.

En las Tablas III y IV (pág. siguiente) se pueden comparar los requerimientos con relación a las características químicas y físicas, respectivamente, de la norma española frente a la normativa internacional.

Al intentar establecer una comparación, hay que tener en cuenta la diferencia de métodos usados para cada especificación, en especial en el caso del Indice de Actividad Puzolánica.

O. Manz (15), por una parte, y E. Rossouw y J. Krüger (16), por otra, presentaron en el Simposio sobre el uso de c.v. en el hormigón celebrado en Canadá en 1983, dos trabajos sobre las especificaciones internacionales de las adiciones para su empleo en el hormigón de cemento portland.

Las dos Tablas citadas, que se ofrecen en este trabajo, se han elaborado teniendo en cuenta estos antecedentes y realizando, además, una actualización de las normas existentes, así como una ampliación en el número de países contemplados, y que, en definitiva, han sido
Tabla II

Relación de normas españolas que figuran en UNE 83415 .

\begin{tabular}{|c|l|}
\hline $\begin{array}{c}\text { NORMA } \\
\text { UNE } \\
\text { N2 }\end{array}$ & "ADICIONES AL HORMIGON. CENIZAS VOLANTES" \\
\hline 83415 & $\begin{array}{l}\text { TITULO } \\
\text { Definición, Especificaciones, Transporte y Almace- } \\
\text { namiento de las Cenizas Volantes utilizadas como } \\
\text { Adición o los Hormigones y Morteros de Ce- } \\
\text { mento Portland. }\end{array}$ \\
\hline 83421 & $\begin{array}{l}\text { Toma, Preparación, Conservación y Transporte } \\
\text { de las Muestras. }\end{array}$ \\
\hline 83434 & $\begin{array}{l}\text { Determinación de la Humedad. } \\
\text { Determinación de Sulfatos expresados en SO z por } \\
\text { Método Gravimétrico. } \\
\text { Determinación de la Pérdida por Calcinación. }\end{array}$ \\
\hline 83450 & $\begin{array}{l}\text { Determinación de la Finuro. } \\
\text { Determinación del Indice de Actividad Resistente } \\
\text { con Cemento Portland. } \\
\text { Demanda de Agua Relativa de una Ceniza Volan- } \\
\text { te mezclada con Cemento Portland en forma de } \\
\text { Mortero. } \\
\text { Determinación de la Estabilidad de Volumen por } \\
\text { el Método Le Chatelier. }\end{array}$ \\
\hline 453 & 452
\end{tabular}

Ios siguientes: Australia (17); Austria (18); Canadá (19); Cembureau (20); Corea (21); España (22); Estados Unidos (23), (24) y (25); Gran Bretaña (26) y (27); India (28); Japón (29); República Democrática Alemana (30); República Federal Alemana (31); Turquía (32); y Rusia (33).

La Norma española UNE 83415 no destaca ni por el número de requerimientos, ni por el valor de los límites de sus especificaciones, tanto en lo que se refiere a los aspectos químicos (Tabla III), como a los físicos (Tabla IV).

En Cataluña se han publicado unas "Recomendaciones para el uso de cenizas volantes en el hormigón" (UC-85) (34) que, entre otros aspectos, indican limitaciones respecto a características químicas y físicas. Los valores recomendados se recogen en la Tabla V.

Para la elaboración de estas Recomendaciones se han tenido lógicamente en cuenta, de un modo especial, las cenizas volantes producidas en la zona catalana. 
Tabla III

Cenizas volantes utilizadas en el hormigón. Requerimientos químicos.

\begin{tabular}{|c|c|c|c|c|c|c|c|c|c|c|c|c|c|c|c|c|c|c|c|}
\hline \multirow{3}{*}{ ESPECIFICACION } & \multirow{3}{*}{ 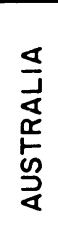 } & \multirow{3}{*}{$\begin{array}{l}\frac{a}{\alpha x} \\
\frac{0}{5} \\
\frac{a}{\alpha}\end{array}$} & \multirow{2}{*}{\multicolumn{2}{|c|}{ 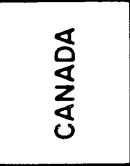 }} & \multirow{3}{*}{ 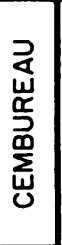 } & \multirow{3}{*}{ 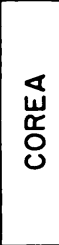 } & \multirow{3}{*}{ 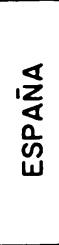 } & \multirow{2}{*}{\multicolumn{2}{|c|}{ 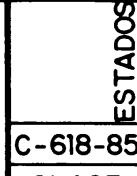 }} & \multicolumn{2}{|c|}{$\begin{array}{l}3 \\
0 \\
2 \\
3\end{array}$} & \multicolumn{2}{|c|}{ 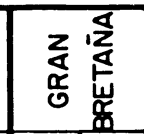 } & \multirow{3}{*}{ 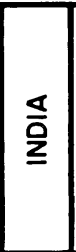 } & \multirow{3}{*}{$\begin{array}{l}z \\
0 \\
\vdots \\
3\end{array}$} & \multirow{3}{*}{ 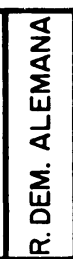 } & \multirow{3}{*}{ 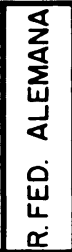 } & \multirow{3}{*}{$\begin{array}{l}\frac{\alpha}{3} \\
0 \\
\frac{x}{2} \\
r\end{array}$} & \multirow{3}{*}{ ஸे } \\
\hline & & & & & & & & & & 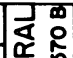 & \begin{tabular}{|l|} 
W \\
os
\end{tabular} & న్ळ & को & & & & & & \\
\hline & & & \multicolumn{2}{|c|}{ CLASE } & & & & \begin{tabular}{|l|} 
CLA \\
$F$
\end{tabular} & & 䇋 & 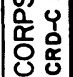 & $\begin{array}{l}\text { m } \\
\text { 邑 }\end{array}$ & 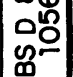 & & & & & & \\
\hline HUMEDAD (máx.\%) & 1,5 & 1 & $\begin{array}{l}3 \\
\text { lopc }\end{array}$ & $\begin{array}{c}3 \\
\text { onal) }\end{array}$ & & 3 & 1,5 & 3 & 3 & 3 & 3 & & 0,5 & & 1 & & & 3 & \\
\hline $\begin{array}{l}\text { PERDIDA POR } \\
\text { CALCINACION (máx.\%) }\end{array}$ & 8 & 5 & 12 & 6 & 5 & 12 & 6 & $\begin{array}{c}6 \\
(12)\end{array}$ & 6 & 6 & 6 & 7 & 7 & 12 & 5 & 15 & 5 & 10 & 10 \\
\hline \begin{tabular}{|c|}
$\mathrm{SiO}_{2}(\min . \%)$ \\
$\mathrm{SiO}_{2}+\mathrm{Al}_{2} \mathrm{O}_{3}+\mathrm{Fe}_{2} \mathrm{O}_{3}(\min \%)$ \\
$(11) \underset{(2)}{(3)}$
\end{tabular} & & $\begin{array}{l}42-60 \\
111 \\
16-32 \\
(2) \\
3-12 \\
(3)^{2} \\
\end{array}$ & & & & 70 & & 70 & 50 & 75 & 70 & & & 70 & 45 & & & 70 & 40 \\
\hline $\mathrm{SO}_{3}$ (máx.\%) & 2,5 & $\begin{array}{l}3,5 \\
\left(+s^{\prime \prime}\right)\end{array}$ & 5 & 5 & & 5 & 4,5 & 5 & 5 & 4 & 4 & 2,5 & 2,5 & 3 & & 3 & 4 & 5 & 3 \\
\hline $\begin{array}{l}\text { CaO (máx.\%) } \\
\text { CoO ibbre (máx.\%) }\end{array}$ & & $\begin{array}{c}5-20 \\
2\end{array}$ & & & 1 & & & & & & & & & & & & & 6 & \\
\hline MgO (máx.\%) & & 5 & & & & 5 & & & & 5 & 5 & 4 & 4 & 5 & & & & 5 & \\
\hline $\begin{array}{c}\text { ALCALIS SOLUBLES } \\
\left(\mathrm{Na}_{2} \mathrm{O} \% \text { móx.) }\right.\end{array}$ & & & & & 1,5 & 1,5 & & $\begin{array}{l}1,5 \\
\text { lopcio }\end{array}$ & $\begin{array}{l}1,5 \\
\text { onal) }\end{array}$ & 2 & 1,5 & & & $\begin{array}{l}1,5 \\
(\mathrm{OPC})\end{array}$ & & & & & \\
\hline OTRAS & & $x$ & & & & & & & & & & & & & & & $x$ & & \\
\hline
\end{tabular}

Tabla IV

Cenizas volantes utilizadas en el hormigón. Requerimientos físicos.

\begin{tabular}{|c|c|c|c|c|c|c|c|c|c|c|c|c|c|c|c|c|c|}
\hline \multirow{3}{*}{ ESPECIFICACION } & \multirow{3}{*}{ 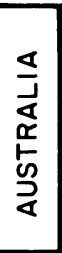 } & \multirow{3}{*}{$\begin{array}{l}\frac{d}{\alpha} \\
\frac{x}{6} \\
\stackrel{a}{\alpha}\end{array}$} & 这 & \multirow{3}{*}{ 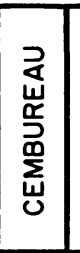 } & \multirow{3}{*}{ 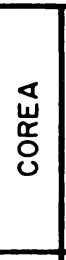 } & \multirow{3}{*}{ 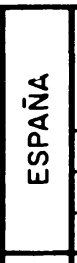 } & \multicolumn{3}{|c|}{ 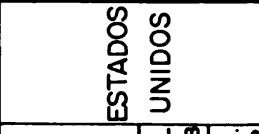 } & \multicolumn{2}{|c|}{ 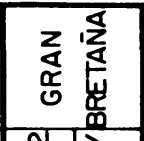 } & \multirow{3}{*}{ ఏ্ড } & \multirow{3}{*}{$\begin{array}{l}z \\
\frac{0}{4} \\
\frac{1}{2}\end{array}$} & \multirow{3}{*}{ 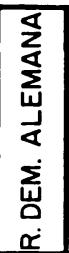 } & \multirow{3}{*}{ 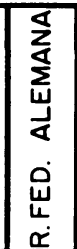 } & \multirow{3}{*}{ 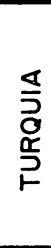 } & \multirow{3}{*}{ 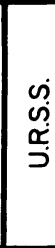 } \\
\hline & & & & & & & $C-618-85$ & $\left.\mid \begin{array}{lll}1 & \infty \\
\hdashline & 0 \\
x & 0 \\
0\end{array}\right]$ & $\mid$ & 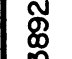 & के & & & & & & \\
\hline & & & \begin{tabular}{l|l}
\multicolumn{3}{c}{ CLASE } \\
P
\end{tabular} & & & & 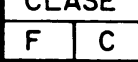 & 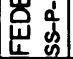 & 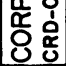 & $\begin{array}{l}m \\
0 \\
0\end{array}$ & 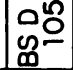 & & & & & & \\
\hline $\begin{array}{l}\text { FINURA } \\
\text { SUPERFICIE ESPECIF. } \\
\text { (cm²/9; móx.) } \\
\text { RESIDUO TAMIZ } \\
\text { (móx. \%) } \\
\text { s } 200 \mu \mathrm{m} \\
\text { s } 90 \mu \mathrm{m} \\
\text { s } 45 \mu \mathrm{m} \\
\mathrm{s} 20 \mu \mathrm{m}\end{array}$ & $\begin{array}{c}10 \\
(150) \\
50\end{array}$ & $\begin{array}{l}4000 \\
5000\end{array}$ & 34 & 2700 & & $\begin{array}{l}15 \\
40\end{array}$ & 34 & & & 12,5 & 12,5 & & 2400 & & $\begin{array}{l}50140 \\
30\end{array}$ & 0,3 & \\
\hline \begin{tabular}{|l} 
INDICE \\
ACTIVIDAD RESISTEN \\
CON CEMENTO(min.\%) \\
7 DIAS \\
28 DIAS \\
91 DIAS \\
CON CAL (min.MPa) \\
7 DIAS \\
\end{tabular} & & 80 & $\begin{array}{c}\overline{8} \\
\stackrel{8}{8} \\
68 \\
75 \text { (op.) }\end{array}$ & 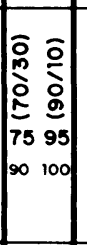 & $\begin{array}{r}85 \\
5,5 \\
\end{array}$ & $\begin{array}{l}0 \\
0 \\
o \\
o \\
75 \\
70 \\
90\end{array}$ & $\begin{array}{l}75 \\
5,5\end{array}$ & 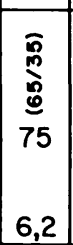 & 6,2 & $856 \mathrm{p}$ & & 3,9 & $\begin{array}{l}60 \\
70\end{array}$ & $x$ & $\begin{array}{l}70 \\
70 \\
70\end{array}$ & 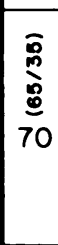 & \\
\hline $\begin{array}{l}\text { DEMANDA DE AGUA } \\
(\text { máx.\%) }\end{array}$ & & & & & 105 & 100 & 105 & & & 95 & 95 & & 102 & & & & \\
\hline $\begin{array}{c}\text { ESTABILIDAD VOLUM. } \\
\text { (máx.) } \\
\text { AUTOCLAVE } \\
\text { OTROS (L.CHAT.) } \\
\end{array}$ & & $x$ & 0,8 & & 0,5 & 10 & 0,8 & 0,5 & 0,5 & & & 0,8 & & & $x$ & 10 & \\
\hline OTRAS & $\bar{x}$ & & $\bar{x}$ & $x$ & $x$ & & $\bar{x}$ & $x$ & $x$ & $\bar{x}$ & & $\bar{x}$ & $x$ & & $\hat{x}$ & $\frac{\pi}{x}$ & \\
\hline
\end{tabular}


Tabla V

Recomendaciones UC-85 Cataluña.

"Uso de las cenizas volantes en el hormigón."

\begin{tabular}{|c|c|c|c|}
\hline $\begin{array}{l}\text { ARTICULO } \\
\text { № }\end{array}$ & $\begin{array}{c}\text { CARACTERISTICAS DE LAS } \\
\text { CENIZAS VOLANTES }\end{array}$ & \multicolumn{2}{|c|}{$\begin{array}{c}\text { VALOR } \\
\text { RECOMENDADO }\end{array}$} \\
\hline 2 & PERDIDA AL FUEGO & \multirow{7}{*}{ 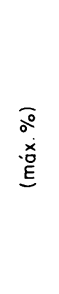 } & 6 \\
\hline 3 & CaO libre & & 7 \\
\hline 4 & FINURA & & \\
\hline & (Residuo en el tamiz de $45 \mu \mathrm{m}$ ) & & 40 \\
\hline 5 & $\mathrm{SO}_{3}$ & & 5 \\
\hline 6 & $\mathrm{MgO}$ & & 5 \\
\hline 7 & HUMEDAD & & $.1,5$ \\
\hline 8 & $\begin{array}{l}\text { INDICE DE PUZOLANICIDAD } \\
\text { (de acuerdo con } \\
\text { BS } 3892: \text { Part } 1 \text { /apéndice F) }\end{array}$ & 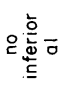 & $85 \%$ \\
\hline
\end{tabular}

\section{CONSIDERACIONES FINALES}

Los primeros pasos en relación a la normativa en España sobre cenizas volantes para su uso en el hormigón, se han dado ya con la aparición de las nuevas normas UNE (Tabla II), avaladas por el conocimiento previo acerca de las características de estos materiales y la experiencia en su utilización.

Las especificaciones contenidas en la Norma UNE 83415 - 82 "ADICIONES AL HORMIGON. CENIZAS VOLANTES: Definición, Especificaciones, Transporte y Almacenamiento de las Cenizas Volantes utilizadas como Adición a los Hormigones y Morteros de Cemento Portland", se encuentran dentro del marco de la normativa internacional existente correspondiente a este tema.

Todo trabajo de normalización requiere una actualización constante. Este hecho se ha tenido en cuenta y todas las normas elaboradas tienen carácter experimental, máxime en esta circunstancia en la que el número de centrales termoeléctricas que se hallan en funcionamiento está en aumento, y las ya antiguas sufren renovaciones y ampliación en sus grupos.

Con el fin de conocer las cenizas volantes que se generan en el tiempo en cada central y poder adecuar, en la medida de lo posible, las normas a los materiales existentes, se está llevando a cabo un Proyecto de Investigación iniciado en 1986 por el Instituto de la Construcción y del Cemento "Eduardo Torroja", bajo la dirección del Prof. F. Soria, y en el que colaboran varios laboratorios de investigación.

En este trabajo se contempla la realización de varios muestreos de cenizas volantes, en fechas sucesivas, de todas las centrales termoeléctricas españolas actuales, y la aplicación de las normas UNE experimentales a cada una de las muestras.

Los resultados obtenidos en estas experiencias podrán servir de base para el establecimiento de las normas españolas con carácter definitivo, aunque siempre abiertas a una revisión posterior, relativas a las cenizas volantes utilizadas como adición a los morteros y hormigones de cemento portland.

\section{AGRADECIMIENTO}

Los autores agradecen al Grupo de Trabajo "Adiciones al Hormigón" de la Comisión Técnica 83 "Hormigones" de IRANOR, actualmente en AENOR, su labor y dedicación en la elaboración de las normas.

\section{BIBLIOGRAFIA}

1. IDORN, G. M. en Effects of Fly Ash Incorporation in Cement and Concrete, Proc. Symposium N (Mat. Res. Soc., University Park, PA), pp $244-259$ (1981).

2. KOVACS, R. y TALABER, J., Silicates Ind., n. 2, pp 31-34 (1984).

3. BUCCHI, R. 8. Congr. Inter. Quim. Cem., Vol. I, pp 185-198, Brasil (1986).

4. JENSEN, S. A., en Fly Ash and Coal Conversion By-ProductsCharacterization, Utilization and Disposal II, edited by G. J. McCarthy, F. P. Glasser and D. M. Roy Mat. Res. Soc., Symp. Proc. Vol. 65, pp 27-36 (1986).

5. COVEY, J. N., Concr. Int., pp 11-15 (1982).

6. PAPAYIANNI, J., Seminario sobre cenizas volantes, pp 65-113, Barcelona (1986).

7. VALENTI, G. L.; CIOFFI, R. y SERSALE, R. en Fly Ash, Silica Fume, Slag and Natural Pozzolans in Concrete, Proc. CANMETIACI 2nd Int. Conf. Madrid, Editor V. M. Malhotra, Vol. 1, pp 741-762 (1986).

8. CALLEJA, J., Cemento-Hormigón, 597 y 598 (1983).

9. LUXAN, M. P.; SANCHEZ de ROJAS, M. I. and SORIA, F. presentado en Fly Ash Silica Fume, Slag and Natural Pozzolans in Concrete, Madrid (1986). Materiales de Construcción IccET (1988, n. ${ }^{\circ}$ 209).

10. DIAMOND, S., Cem. Conc. Res., 16, 3, pp 569-579 (1986).

11. MEHTA, P. K. en The Use of Fly Ash, Silica Fume, Slag and Other Mineral by-Products in Concrete, Proc. CANMETIACI, First Int. Conf. Montebello, Editor V. M. Malhotra, Vol.1. pp 1-46 (1983).

12. MEHTA, P. K., Cem. Concr. Res., 15, pp 669-74 (1985). 
13. LUXAN, M. P.; SANCHEZ de ROJAS, M. I., presentado al Symposium N, "Fly Ash and Coal Conversion By-Products: Characterization, Utilization and Disposal III, Boston (1986)

14. CABRERA, J. y col. en Fly Ash, Silica Fume, Slag and Natural Pozzolans in Concrete, Proc. CANMET/ACI 2nd Int. Conf. Madrid, Editor V. M. Malhotra, vol 1, pp 115-144 (1986).

15. MANZ, O. E. en The Use of Fly Ash, Silica Fume, Slag and Other Mineral by-Products in Concrete, Proc. CANMET/ACI, First Int. Conf. Montebello, Editor V. M. Malhorta, vol 1, pp 187-200 (1983).

16. ROSSOUW, E.; KRÜGER, J. en The Use of Fly Ash, Silica Fume, Slag and Other Mineral by-Products in Concrete, Proc. CANMET/ACI, First Int. Conf. Montebello, Editor V. M. Malhotra, vol 1, pp 201-220 (1983).

17. Standards Association of Australia "Fly Ash for use in concrete". AS 1129 y 1130 (1971).

18. Österreichisches Normungsinstitut. "Draft Specification for Fly Ash for Concrete Production" B 3320-1981.

19. Canadian Standards Association. "Suplementary Cementing Materials and their use in Concrete Construction". CAN 3-A23.5 - M82 (1982).

20. CEMBUREAU. Report to Cembureau Technical Committee "Specifications for fly ash as a Concrete additive" (1982).

21. Korean Industrial Standard. "Fly Ash for use as an Admixture in Portland Cement Concrete". KS L 5405 (1964).

22. Norma Española UNE 83 415: "Adiciones al Hormigón. Cenizas Volantes: Definición, Especificaciones, Transporte y Almacenamiento de las Cenizas Volantes utilizadas como Adición a los Hormigones y Morteros de Cemento Portland" (1986).
23. American Society for Testing Materials. "Standard Specification for Fly Ash and Raw or Calcined Natural Pozzolan for Use as a Mineral Admixture in Portland Cement Concrete", ASTM C 618-85 (1985).

24. Federal Specification, "Pozzolan for use in Portland Cement Concrete". SS-P-570 B, April 1969.

25. U.S. Army Corps of Engineers. "Pozzolan for use in Portland Cement Concrete". CRD - C 262-63.

26. British Standards Institution. "Pulverized-Fuel Ash. Part 1. Specificacion for pulverized-fuel ash for use as a cementitious component in structural concrete." BS 3892 (1982).

27. British Standards Institution. "Draft British Standard Specification for Pulverized-Fuel Ash for use in Concrete." BS Draft $81 / 10567$.

28. Indian Standards Institution. "Standard Specification for Fly Ash. (Part 2: For use as Admixture for Concrete)." IS: 3812 (Part 2). (1966).

29. Japanese Industrial Standard. "Fly Ash”. JIS A 6201 - (1977).

30. Deutsche Normen. TGL 28 101/03.

31. Deutsche Normen. DIN 1045-1980. Richtlinie für die Erteilung von Prüfzeichen für Steinkohlenflugasche als Betonzusatzstoff nach DIN 1045 Bautechnik (11), 39, (1980).

32. Turkish Standards Institution. "Fly Ashes suitable for Mixing with Portland Cement Concrete." TS 639-1968.

33. GOST. "Binder Active Mineral Additives." GOST 6269-63.

34. Recomendaciones UC-85: "Uso de las cenizas volantes en el hormigón", D.O.G. n. 534, (3-V-85). 